\title{
A NOTE ON A FUNCTIONAL EQUATION
}

\author{
CHUNG-MING AN \\ (Received 4 February 1970) \\ Communicated by B. Mond
}

The object of this note is to give an aspect to the problem of the functional equation of the generalized gamma function and Dirichlet series which are defined in [1]. ${ }^{1}$ In general, we cannot answer the problem yet. But it is worthy to attack this problem for some special cases.

Throughout this note $\mathbb{R}$ and $Z$ denote the reals and the integers respectively. Let $F(X)$ and $E(X)$ be two homogeneous polynomials in $\mathbb{R}[X], X=\left(X_{1}, \cdots, X_{n}\right)$, of degree $d>0$. Asume that $F(x) \neq 0, E(x) \neq 0$ for non-zero $x \in \mathbb{R}^{n}$. We put

$$
\begin{aligned}
\zeta(F, s) & =\sum_{\gamma \in Z^{n-\{0\}}} F(\gamma)^{-s}, \\
\Gamma(E, s) & =\int_{\mathbb{R}^{n}} e^{-|x|^{2}} E(x)^{s-(n / d)} d x, \\
\xi(E, F, s) & =\pi^{-(d s) / 2} \Gamma(E, s) \zeta(F, s),
\end{aligned}
$$

where $|x|^{2}=x_{1}^{2}+\cdots+x_{n}^{2}$ and $d x=d x_{1} \cdots d x_{n}$. It is proved in [1] that $Z(F, s)$ and $\Gamma(E, s)$ are meromorphic functions of $s$.

If $n=1$, we may assume $F(X)=a X^{d}, a>0$, and put $F^{-1}(X)=(1 / a) X^{d}$. We denote the number $a$ by $|F|$. We shall use the same notations for $E(X)$.

If $n \geqq 2$, we only consider the case of the quadratic forms, i.e., $d=2$. Let

$$
E(X)=\sum_{i, j=1}^{n} e_{i j} X_{i} X_{j}, \quad F(X)=\sum_{i, j=1}^{n} f_{i j} X_{i} X_{j}
$$

be two positive-definite quadratic forms. We may assume that $E=\left(e_{i j}\right)$ and $F=\left(f_{i j}\right)$ are two $n \times n$ positive-definite matrices with real entries. So $|E|=\operatorname{det}(E)$ $\neq 0,|F|=\operatorname{det}(F) \neq 0$. Let $E^{-1}$ and $F^{-1}$ be the inverse matrices of $E$ and $F$, respectively.

1 The result in [1] appeared in the Bulletin of the American Mathematical Society, May 1969. 
The main result of this paper is

THEOREM. Under the above assumptions, $\xi(E, F, s)$ satisfies the following functional equation

$$
\xi\left(E^{-1}, F^{-1}, \frac{n}{d}-s\right)=|E|^{\frac{1}{2}},|F|^{\frac{1}{2}}, \xi(E, F, s) .
$$

For $n=1$, we observe

$$
\begin{aligned}
& \zeta(F, s)=a^{-s}\left(1+K^{-s}\right) \zeta(d s) \\
& \Gamma(E, s)=a^{s-n / d}\left(1+K^{s-n / d}\right) \Gamma\left(\frac{d s}{2}\right)
\end{aligned}
$$

where $K=(-1)^{d}, \xi(s)$ is the Riemann-zeta function and $\Gamma(s)$ is the gamma function. Then, the theorem for $n=1$ follows immediately from the functional equation of the Riemann zeta function.

For $n \geqq 2$ and $d=2$, we shall use the polar coordinates in $n$-dimension ([1]). Thus, we have

$$
\Gamma(E, s)=\frac{1}{2} \Gamma(s) \int_{S^{n-1}} E(\omega)^{s-n / 2} d \omega .
$$

We want to prove the following lemma:

LEMMA. If $n \geqq 2$ and $d=2$, then

$$
|E|^{\frac{1}{2}} \int_{S^{n-1}} E(\omega)^{s-n / 2} d \omega=\int_{S^{n-1}} E^{-1}(\omega)^{-s} d \omega .
$$

Proof. It is well known that there is an orthogonal $n \times n$ matrix $U$, i.e., $U^{-1}={ }^{t} U$, such that

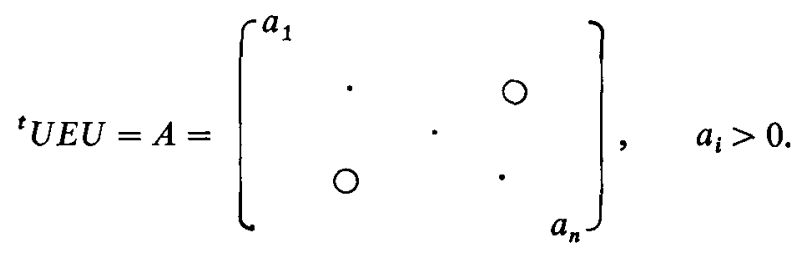

Viewing $X$ as a $n \times 1$ matrix, $E(X)={ }^{r} X E X$ and changing variables by $X=U Y$, we shall have $d x=d y, E(X)=A(Y)$ and $|x|^{2}=|y|^{2}$. Hence, $\Gamma(E, s)=\Gamma(A, s)$, i.e.,

$$
\int_{S^{n-1}} A(\omega)^{s-n / 2} d \omega=\int_{S^{n-1}} E(\omega)^{s-n / 2} d \omega .
$$

Thus, it is enough to prove the lemma for the matrix A. 
Let

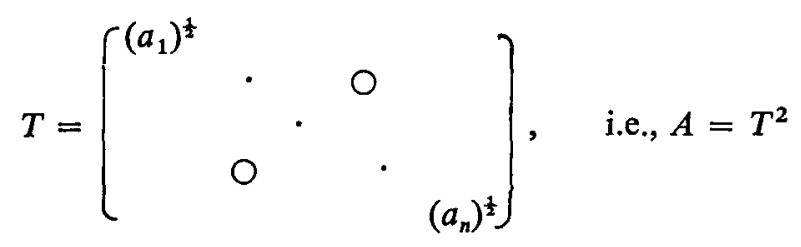

and $z=T y$. We shall have $d y=|T|^{-1} d z, A(y)=|z|^{2}$ and $A^{-1}(z)=|y|^{2}$. So

$$
\Gamma(A, s)=|A|^{-\frac{1}{2}} \int_{\mathbb{R}^{n}} e^{-A^{-1}(z)}|z|^{2 s-1} d z .
$$

By changing variables into the polar coordinates, i.e., $z=r \omega$, we get

$$
\Gamma(A, s)=|A|^{-\frac{1}{2}} \int_{S^{n-1}} \int_{0}^{\infty} e^{-r^{2} A^{-1}(w)} r^{2 s-1} d r d \omega
$$

Put

$$
H(\omega)=\int_{0}^{\infty} e^{-r^{2} A^{-1}(w)} r^{2 s-1} d r .
$$

Since $A^{-1}(\omega)>0$, for all $\omega \in S^{n-1}$, we may put $t=\left(A^{-1}(\omega)\right)^{\frac{1}{2}} r$.

Then

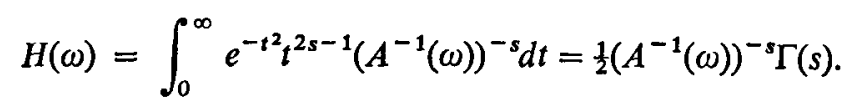

So, we shall have

$$
\Gamma(A, s)=\frac{1}{2}|A|^{-\frac{1}{2}} \Gamma(s) \int_{S^{n-1}}\left(A^{-1}(\omega)\right)^{-s} d \omega .
$$

But, on the other hand

$$
\Gamma(A, s)=\frac{1}{2} \Gamma(s) \int_{S^{n-1}}(A(\omega))^{s-\frac{1}{2} n} d \omega .
$$

From above two forms, it is clear to see the lemma. q.e.d.

If we apply the functional equation for Epstein zeta function, i.e., ([2]),

$$
|F|^{\frac{1}{2}} \pi^{-s} \Gamma(s) \zeta(F, s)=\pi^{-\left(\frac{1}{2} n-s\right)} \Gamma\left(\frac{1}{2} n-s\right) \zeta\left(F^{-1}, \frac{1}{2} n-s\right)
$$

and the lemma to $\xi(E, F, s)$, we shall obtain

$$
\xi\left(E^{-1}, F^{-1}, \frac{1}{2} n-s\right)=|E|^{\frac{1}{2}}|F|^{\frac{1}{2}} \xi(E, F, s),
$$

which proves the theorem. 
For general cases, I think that all the difficulties in solving this problem of the functional equation come from the integral over $n$-sphere

$$
\int_{S^{n-1}} E(\omega)^{s-n / d} d \omega
$$

in the form of the generalized gamma function and from the lack of theta-formula for the polynomials of higher degree. For example, we may define the function

$$
\theta(\tau, x)=\sum_{\gamma \varepsilon Z^{n}} \exp (2 \pi i \tau F(\gamma+x))
$$

for a positive-definite homogeneous polynomial $F(X) \in \mathbb{R}[X]$. But, the information for non-quadratic forms is inadequate. Some work in this direction has been done by Ekkehard Krätzel [3].

\section{References}

[1] Chung-ming An, On a generalization of gamma function ant its application to certain Dirichlet series, Dissertation, (University of Pennsylvania, 1969).

[2] C. L. Siegel, Lecture on advanced analytic number theory, (Tata Institute of Fundamental Research, Bombay, India, 1961).

[3] Ekkehard Krätzel, Höhere theta funslionen; I, II. Math. Nachr: 30 (1965), 17-46.

Department of Mathematics

The Johns Hopkins University

Baltimore, Maryland 